\title{
Design of Gracefully Degradable Hypercube-Connected Systems*
}

\author{
Tze Chiang LeE \\ IBM Corporation, T. J. Watson Research Center, P.O. Box 704, Yorktown Heights, New York 10598 \\ JoHN P. HAYES

\begin{abstract}
Advanced Computer Architecture Lab, Electrical Engineering \& Computer Science Department, University of Michigan,
\end{abstract} \\ Ann Arbor, Michigan 48109-2122
}

\begin{abstract}
We address the problem of modifying a hypercube computer by the addition of spare nodes and links to improve its fault tolerance, while maintaining a specified level of performance. The hypercube is modeled by a graph in which nodes represent processors and edges represent communication links. A new graphbased measure of performance degradation is introduced. This characterizes a fault-tolerant hypercube as $k$-fault-tolerant $(k-\mathrm{FT})$ $g$-step-degradable $(g-S D)$ if the removal of any $k$ nodes reduces the dimension of the largest fault-free subcube by at most $g$. We show how to construct $k$-FT $g$-SD hypercubes for values of $k$ up to 16 and $g=0,1$, or 2. Many of these designs are shown to be link- or degree-optimal. We also propose a construction method that uses small $k$-FT $g$-SD designs as seeds to construct $k$-FT $g$-SD designs of larger sizes. This results in fault-tolerant hypercubes in which reconfiguration can be first done locally and then easily extended to the entire system. The small number of added links and nodes is shown to be useful not only in increasing the fault tolerance of the underlying hypercube, but also in reducing the average internode distance. 1992 Academic Press, Inc.
\end{abstract}

\section{INTRODUCTION}

Since hypercube architectures are very regular and scalable, programs for these computers are frequently designed with the dimension $n$ of the host hypercube $Q_{n}$ treated as an input variable, so that the same program can be run without modification on hypercubes of different sizes. This scalability property of hypercube architecture makes it possible to tolerate faults gracefully by confining a program to a fault-free subcube of the host hypercube. The reduction of the effective cube size due to faults is a rough indication of the performance degradation. Based on this observation, we present a new characterization of fault-tolerant hypercube architectures that allows the performance degradation due to faults to be measured. We also develop some methods to modify $Q_{n}$ by the addition of spare nodes and communication links in order to obtain designs whose performance degrades gracefully in the presence of node failures.

\footnotetext{
* This work was supported by the Office of Naval Research under Contracts N00014-85K0531 and N00014-90J1860.
}

Becker and Simon [2] have discussed the problem of finding the minimum number of faults needed to destroy every fault-free subcube $Q_{k}$, in $Q_{n}$, and give some analytic bounds on these numbers. Graham et al. [6] have improved the bounds and obtained exact values for some cases. These results show that for $n>k+2, Q_{n}$ is a good fault-tolerant realization of $Q_{k}$. For example, the numbers of faults required to destroy every fault-free $Q_{n-3}$ and $Q_{n-4}$ in $Q_{n}$, for $n \geq 6$, are at least 12 and 24 , respectively. However, in the worst case, two faults in antipodal positions destroy every fault-free $Q_{n-1}$ and thus degrade the maximum performance of $Q_{n}$ by a factor of 4 . This leads to an interesting question which is the subject of this paper: How can $Q_{n}$ be modified efficiently so that its fault tolerance, measured by the number of faults required to destroy every fault-free $Q_{k}$, is increased?

We define $Q(n, k, g)$ to be a graph that contains $Q_{n}$ and is such that the removal of any $k$ nodes from it results in a graph containing $Q_{n-g}$. It is notationally convenient to use $Q(n, k, g)$ to denote the class of graphs of interest, as well as specific members of this class. The parameter $k$ is the measure of fault tolerance, and we introduce the $d i$ mension loss $g$ to measure the performance degradation. A $Q(n, k, g)$ is then termed a $k$-fault-tolerant $g$-stepdegradable ( $k$-FT $g$-SD) $Q_{n}$. The fault-tolerance design problem for hypercubes can now be stated as follows: given $n, k$ and $g$, find a $Q(n, k, g)$ whose cost is a minimum. This cost may be measured by the number of nodes or links of $Q(n, k, g)$, or by its maximum node degree. Since $Q_{n}$ itself is a good realization of a $Q(n, k, g)$ for $n \gg$ $g \geq 3$, the cases where $g \leq 2$ are of most interest.

Previous research $[1,11,12]$ on fault-tolerant hypercube design has, in terms of our notation, only considered the problem of constructing $Q(n, k, 0)$. Most of the proposed designs, however, suffer from excessive maximum node degrees. In this paper, we study the design of $Q(n, k, g)$, where $0 \leq g \leq 2$. We construct $Q(n, k, g)$ by adding a small, preferably minimum, number of extra nodes and links to $Q_{n}$. If a design with a minimum number of nodes and links is not attainable, near-optimal solutions are sought. Another concern is to minimize the 
maximum node degree of the modified hypercube. In a real computer system, the degree of a node represents the number of communication channels attached to it, and can significantly affect system cost. Furthermore, the degree of a node is limited by packaging constraints. Hence, in addition to minimizing the number of nodes and links, we also consider minimizing the maximum node degrees in our designs.

To facilitate the discussion, the following standard notation is used [7]. The minimum degree among the nodes of $G$ is denoted by $\delta(G)$, while the largest node degree is denoted by $\Delta(G)$. If all nodes have the same degree, i.e., $\delta(G)=\Delta(G)=r$, then $G$ is called regular of degree $r$. The connectivity $\kappa=\kappa(G)$ of a graph $G$ is the minimum number of nodes whose removal results in a disconnected or trivial graph.

\section{ZERO-STEP-DEGRADABLE DESIGNS}

Yanney [12] presents several fault-tolerant hypercube designs which, in our terminology, correspond to $Q(n, k$, 0) graphs. One of his approaches is based on the use of global spares, but unfortunately, is not appropriate for the fault-tolerant design of a large graph since it requires that the global spares connect to every node in the graph. It therefore results in an excessively high node degree for the spares. Renncls [11] has proposed the implementation of a global sparing method at the subcube level and the use of crossbar switches to reduce node degree. Yanney [12] also considers the use of local spares. A local spare can only be used to replace a subset of nodes in the graph. A fault-tolerant hypercube can be constructed if every node in the hypercube can be replaced by some spare node(s). However, this approach is also not appropriate for higher-dimensional hypercubes, since the maximum node degree is still intolerably high.

Banerjee et al. [1] develop a different $Q(n, 1,0)$ design, for $n \geq 2$. This design uses $2^{n-2}$ spares, where one spare is added to each of the $2^{n-2}$ disjoint $Q_{2}$ 's. Although its hardware overhead is higher $(25 \%)$ than designs using global spares, the spares in this structure increase the maximum node degree by only 2 . However, this design is restricted to the 1-FT case.

In the following, we describe our solutions to the $Q(n$, $k, 0)$ design problem. The designs of $Q(n, k, 0)$ for small $n$ are obtained by using power graphs of loops. Then we propose a systematic construction method that uses $Q(n, k, 0)$ with small $n$ as a "seed"' to construct $Q(n, k, 0)$ for large $n$. This construction method is based on the product operation on graphs, and can be used for $Q(n, k$, $g$ ), where $g>0$, as well. The maximum node degrees of our designs are relatively small compared to those of previous designs.

\subsection{Minimum Node Designs}

We now present several promising $Q(n, 1,0)$ designs for small $n$. Figure 1 shows designs for $Q(1,1,0), Q(2,1$, $0), Q(3,1,0)$, and $Q(4,1,0)$, all of which use the minimum number of spare nodes. A $Q(n, k, 0)$ with the minimum number of spares is degree-optimal if the maximum node degree is the minimum possible. It can be easily seen that Fig. 1a is a degree-optimal $Q(1,1,0)$ design. In general, the complete $r$-node graph $K_{r}$ is a degree-optimal $Q(1, r-2,0)$, for $r \geq 3$.

The foregoing $Q(n, k, 0)$ designs can all be interpreted as power graphs of loops (cycles). The $m$ th power graph $G^{m}$ of $G$ is constructed by adding edges to $G$ such that every node $x$ is connected to all nodes at distance $d$ from $x$, for $m \geq d \geq 2$. Let $C_{n}$ denote a loop with $n$ nodes. Figures 1a, 1b, 1c, and $1 \mathrm{~d}$ demonstrate $C_{3}^{1}, C_{5}^{2}, C_{9}^{3}$, and $C_{17}^{5}$, respectively. Our use of power graphs of loops as fault-tolerant designs of $Q_{n}$ is based on two facts: (1) $Q_{n}$ is a subgraph of $C_{2^{n}}^{2^{n-2}}$; and (2) removing a node from $C_{2^{n}+1}^{2^{n}-2+1}$ results in a graph containing $C_{2^{n}}^{2^{n-2}}$, and hence containing $Q_{n}$. Furthermore, the removal of any $k$ nodes from $C_{2^{n}+k}^{2^{n-2}+k}$ results in a graph containing $C_{2^{n}}^{2^{n-2}}$.

THEOREM 1. $\quad C_{2^{n+k}}^{2^{n-2}+k}$ is a $Q(n, k, 0)$ design.

The reconfiguration of these $Q(n, k, 0)$ designs in the presence of faults is fairly simple. Suppose that we have a $C_{2^{n+k}}^{2^{n-2}+k}$ with $t$ faults, where $t \leq k$. Let the nonfaulty nodes be labeled clockwise from 0 to $2^{n}+k-t-1$. The subgraph composed of nodes 0 to $2^{n}-1$ contains $C_{2^{n}}^{2^{n-2}}$.

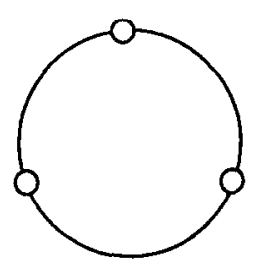

(a)

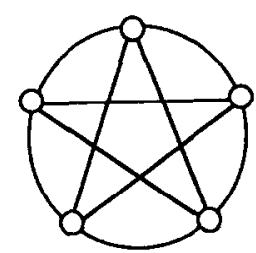

(b)

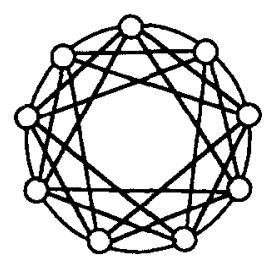

(c)

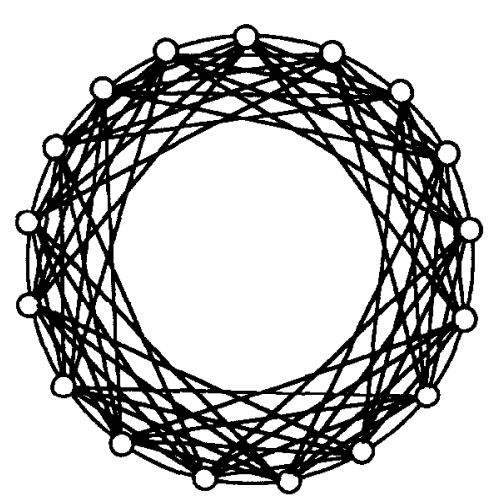

(d)

FIG. 1. The $Q(n, 1,0)$ graphs: (a) $C_{3}^{1}$, (b) $C_{5}^{2}$, (c) $C_{9}^{3}$, and (d) $C_{17}^{5}$. 


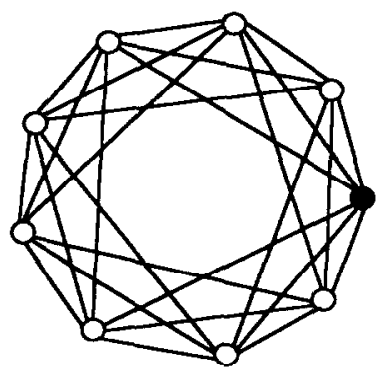

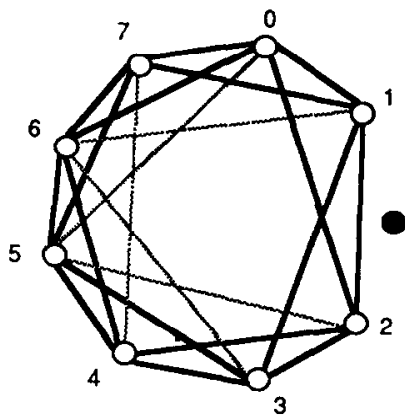

(b)

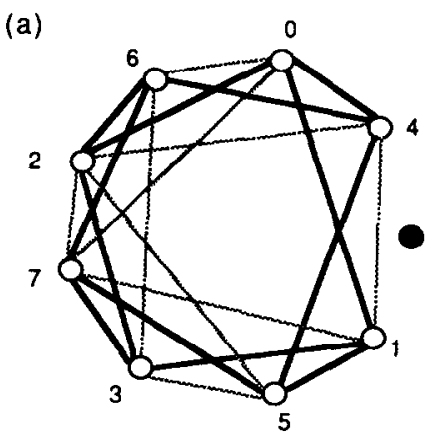

(c)
FIG. 2. (a) Proposed $Q(3,1,0)$ design with a faulty node. (b) Reconfigured $C_{8}^{3}$. (c) Fault-free $Q_{3}$.

Now we relabel each node $x$ to construct a $Q_{n}$ in $C_{2^{n}}^{2^{n-2}}$. Let $x=i \times 2^{n-2}+m$, for some $m \leq 2^{n-2}$. For each node $x$ in $C_{2^{n}}^{2^{n-2}}, x$ is relabeled as $2^{m+1}+y$, if $m \neq 0$, or as $y$, if $m=$ 0 , where $y$ is $0,1,3$, and 2 when $i$ is $0,1,2$, and 3 , respectively. To illustrate, Fig. 2a shows $C_{9}^{3}=Q(3,1,0)$, with a faulty (black) node present. Following the procedure described above, $C_{8}^{2}$ is recovered as shown in Fig. 2b. A fault-tree $Q_{3}$ is obtained accordingly by relabeling the nodes and is depicted in Fig. 2c.

Dutt and Hayes propose similar minimum node $Q(n, k$, 0 ) designs using "circulant" graphs [5]. The node degree of their design is similar to ours when $n$ is small, but is smaller when $n$ becomes larger. This reduction in node degree is, however, at the cost of a more complicated reconfiguration process. In the following, we describe a construction method that effectively reduces the required node degree without increasing the complexity in reconfiguration.

\subsection{Graph-Product Method}

Using the power graph of a loop as a fault-tolerant realization of a hypercube has the merit that the resultant graph is regular, and only simple reconfiguration is needed to recover a fault-free $Q_{n}$. The number of spare nodes used is also a minimum. However, the maximum node degree increases quickly with the size of hypercubc. For example, the 1-FT 0-SD realization of $Q_{6}$ by $C_{65}^{17}$ has node degree 34 . This may make the design impractical for large hypercubes. To address this problem,

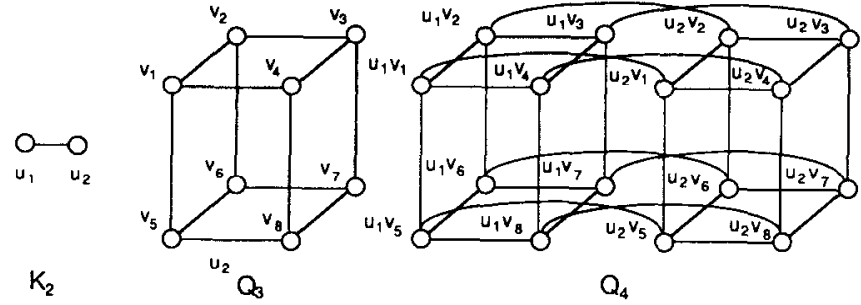

FIG. 3. The graph $Q_{4}$ as the product of $K_{2}$ and $Q_{3}$.

we introduce below a construction method that greatly reduces the maximum node degree by using more spare nodes than the minimum. Specifically, we show that $Q(n+1, k, 0)$ can be constructed systematically from the product graph of $K_{2}$ and $Q(n, k, 0)$. This novel construction method is significant since it allows us to extend designs of smaller $n$, which are usually quite simple, to large $n$. Furthermore, as is shown later, fault-tolerant designs constructed by this method can be easily reconfigured when faults occur. This construction method also applies to more general $Q(n, k, g)$ designs, which are discussed in later sections.

The product of $G_{1}$ and $G_{2}$ is a graph $G_{1} \times G_{2}$ with a node set $V$. Two nodes $u=\left(u_{1}, u_{2}\right)$ and $v=\left(v_{1}, v_{2}\right)$ in $V$ are adjacent if $u_{1}=v_{1}$ and $u_{2}$ is adjacent to $v_{2}$ in $G_{2}$, or if $u_{2}=v_{2}$ and $u_{1}$ is adjacent to $v_{1}$ in $G_{1}$. Figure 3 shows the product of $K_{2}$ and $Q_{3}$. The resulting graph is, by definition, $Q_{4}$. The graph-product construction is characterized in the following theorem.

Theorem 2. Suppose that $|G| \leq 2^{n+1}$. $G^{\prime}=K_{2} \times G$ is a $Q(n+1, k, 0)$ design if and only if $G$ is a $Q(n, k, 0)$ design, for $n, k \geq 2$.

Proof. (1) If: Let $G^{\prime}$ be as shown in Fig. 4, where $G_{1}$ and $G_{2}$ are two identical copies of $G$. Let nodes in $G_{1}$ be denoted by $x_{1}, x_{2}, \ldots$, and $x_{m}$, and nodes in $G_{2}$ be denoted by $x_{1}^{\prime}, x_{2}^{\prime}, \ldots$, and $x_{m}^{\prime}$. Node $x_{i}$ is connected to node $x_{i}^{\prime}$, for $i$ from 1 to $m$. Suppose that the faulty node set is $\left\{x_{f_{1}}, x_{f_{2}}\right.$,

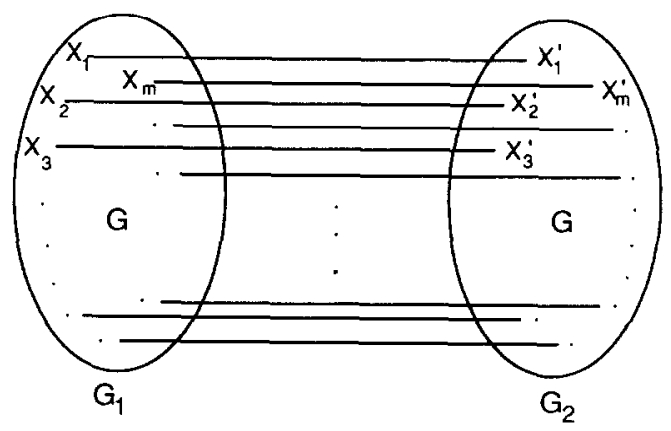

FIG. 4. $G^{\prime}$, a graph constructed as two copies of $G$ 
$\left.\ldots, x_{f_{j}}\right\} \cup\left\{x_{f_{j+1}}^{\prime}, x_{f_{j+2}}^{\prime}, \ldots, x_{f_{k}}^{\prime}\right\}$. Now we generate a "pseudo-fault" set $\left\{x_{f_{1}}, x_{f_{2}}, \ldots, x_{f_{j}}\right\} \cup\left\{x_{f_{j+1}}, x_{f_{j+2}}, \ldots, x_{f_{k}}\right\}$, where $\left\{x_{f_{j+1}}, x_{f_{j+2}}, \ldots, x_{f_{k}}\right\}$ is the set of neighbors of $\left\{x_{f_{j+1}^{\prime}}^{\prime}\right.$, $\left.x_{f_{j+2}}^{\prime}, \ldots, x_{f_{k}}^{\prime}\right\}$ in $G_{1}$. Since $G$ is a $Q(n, k, 0)$ graph, we can always recover a $Q_{n}$ from $G_{1}$ so that none of the nodes in the recovered $Q_{n}$ belong to the pseudo-fault set. Also, in consequence of the definition of the pscudo-fault set, the neighbors in $G_{2}$ of the recovered $Q_{n}$ in $G_{1}$ are nonfaulty. Thus combining the two $Q_{n}$ 's, we obtain a fault-free $Q_{n+1}$ in $G^{\prime}$ for the given $k$ faults.

(2) Only if: Suppose that $G_{1}$ is not a $Q(n, k, 0)$ graph. We show that $k$ faults can destroy every fault-free $Q_{n+1}$ in $G^{\prime}$. We first find a fault set $F_{1}=\left\{x_{1}, x_{2}, \ldots, x_{k}\right\}$ that destroys every fault-free $Q_{n}$ in $G_{1}$. We can specify a second fault set $F_{2}=\left\{a_{1}, a_{2}, \ldots, a_{i}, a_{i+1}^{\prime}, \ldots, a_{k}^{\prime}\right\}$, where $a_{j}=x_{j}$ and $a_{l}^{\prime}=x_{l}^{\prime}$, for some $i, 1 \leq j \leq i<l \leq k . F_{2}$ is obtained by moving some of the nodes in $F_{1}$ from $G_{1}$ to their direct neighbors in $G_{2}$. With fault set $F_{2}$ present, there can be no fault-free $Q_{n+1}$ in either $G_{1}$ or $G_{2}$ since each contains only $2^{n+1}$ nodes. There is also no fault-free $Q_{n+1}$ consisting of a fault-free $Q_{n}$ in $G_{1}$ combined with a fault-free $Q_{n}$ in $G_{2}$.

We claim that there are no other configurations of fault-free $Q_{n+1}$ 's in $G^{\prime}$ under fault set $F_{2}$. To prove this, first suppose that there exists a fault-free $Q_{n+1}$ in the faulty $G^{\prime}$ that consists of a set $S_{1}$ of $i$ nodes in $G_{1}$, and a set $S_{2}$ of $2^{n+1}-i$ nodes in $G_{2}$. Neither $S_{1}$ nor $S_{2}$ forms a $Q_{n}$. Each node in $S_{1}$ has at most one link connected to $S_{2}$, since each node in $G_{1}$ has only one link connected to $G_{2}$. At least two nodes in $S_{1}$ are connected to nodes in $S_{2}$ for $n \geq 2$, since $Q_{n}$ is Hamiltonian.

Now we show that every node in $S_{1}$ must have one link connected to a node in $S_{2}$. Suppose that this is not true. It is easy to find a node $x$ in $S_{1}$ that connects to a node $x^{\prime}$ in $S_{2}$, and has a neighbor $x_{1}$ in $S_{1}$ that has no neighbor in $S_{2}$. Let the dimension between $x$ and $x^{\prime}$ be $m$, and that between $x$ and $x_{1}$ be $j$. We can also find a node $x_{1}^{\prime}$ in $S_{2}$ connecting to $x^{\prime}$ along dimension $j$, hence $x^{\prime}, x, x_{1}$, and $x_{1}^{\prime}$ must form a $Q_{2}$. This violates the assumption that $x_{1}$ has no neighbor in $S_{2}$. Hence each node in $S_{1}$ must have a neighbor in $S_{2}$. This fact also implies $\left|S_{1}\right|=\left|S_{2}\right|=2^{n}$.

Again assume that $x$ in $S_{1}$ connects to node $x^{\prime}$ in $S_{2}$ along dimension $m$. Since neither $S_{1}$ nor $S_{2}$ forms a $Q_{n}$ and each node in $S_{1}$ connects to a node in $S_{2}$, we can find a neighboring node $x_{1}$ of $x$ in $S_{1}$ such that $x_{1}$ connects to $x$ along dimension $j$, and $x_{1}$ connects to node $x_{1}^{\prime}$ in $S_{2}$ along a dimension other than $m$ and $j$, say $l$. Let $x_{2}$ be the neighboring node of $x$ in $S_{1}$ along dimension $l$. It is apparent that $x_{2}$ should connect to $x_{1}^{\prime}$ along dimension $j$. But $x_{1}^{\prime}$ is already connected to $x_{1}$, which leads to a contradiction. Hence $S_{1}$ and $S_{2}$ must form $Q_{n}$ 's and $G_{1}$ and $G_{2}$ are required to be $Q(n, k, 0)$ graphs.

This theorem implies that we can use $Q(n, k, 0)$ designs of small $n$ as seeds to build up $Q(n, k, 0)$ designs of large $n$. Figure $5 \mathrm{a}$ illustrates a $Q(4,1,0)$ that is constructed

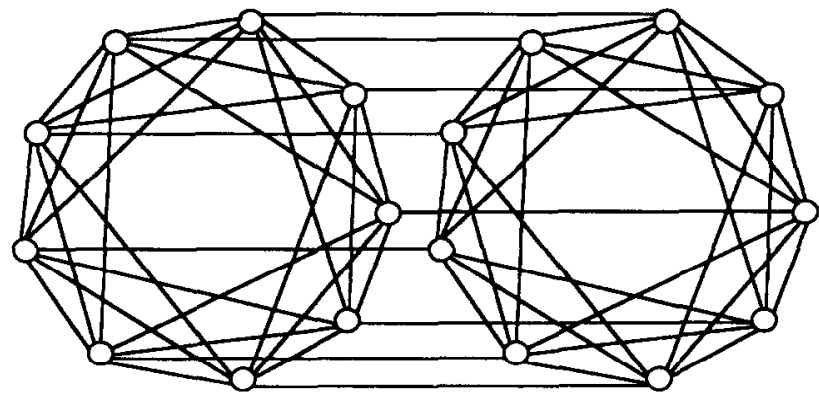

(a)

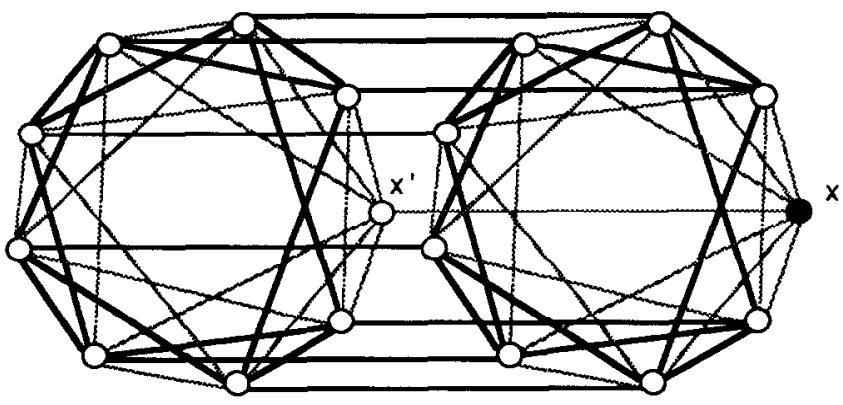

(b)

FIG. 5. (a) A $Q(4,1,0)$ constructed as the product of $K_{2}$ and $Q(3,1,0)$. (b) A recovered fault-free $Q_{4}$ in the presence of a faulty node $x$.

using $Q(3,1,0)$ taken from Fig. 1c as a seed. Larger designs are obtained by applying the graph-product operations several times. Suppose that a $Q\left(n_{1}, k, 0\right)$ is used as a seed and its maximum node degree is $d$. The maximum node degree of a $Q(n, k, 0)$ produced by the foregoing construction method is then $d+n-n_{1}$, which is considerably smaller than that of $Q(n, k, 0)$ developed using other methods. The number of nodes and links of the proposed designs and some of their topological properties are listed in Table I. As can be seen, the designs span a broad spectrum with different amounts of redundancy and maximum node degrees.

Another advantage of the $Q(n, k, 0)$ designs constructed by the graph-product operation is that reconfiguration around faults is fairly simple and efficient. It can first be done locally within a seed and then applied to the whole structure. Suppose that $F=\left\{x_{1}, x_{2}, \ldots, x_{j}\right\}$ is the fault set. We generate a pseudo-fault set $F^{\prime}$ by projecting the faults into a seed $Q\left(n_{1}, k, 0\right)$. Taking the case in Fig. $5 \mathrm{~b}$ as an example, the product graph $Q(4,1,0)$ contains two copies of $Q(3,1,0)$ which serve as seeds. We project the faulty node $x$ into the left seed and create a pseudo-fault $x^{\prime}$. Now we can easily find a pseudo-faulttree $Q_{3}$ in that seed using the method described in Section 2.1. It is clear that the corresponding $Q_{3}$ in the other seed of the $Q(4,1,0)$ is also fault-free. Together, the two $Q_{3}$ 's form a fault-free $Q_{4}$. 
TABLE I

Topological Properties of Some $Q(n, k, 0)$ Designs Constructed by the Graph Product Method

\begin{tabular}{cccccc}
\hline Graph & Seed & $\begin{array}{c}\text { Number } \\
\text { of nodes }\end{array}$ & $\begin{array}{c}\text { Maximum } \\
\text { node degree }\end{array}$ & Diameter & $\begin{array}{c}\text { Number } \\
\text { of links }\end{array}$ \\
\hline$Q(n, 1,0)$ & $Q(1,1,0)$ & $2^{n}+2^{n-1}$ & $n+1$ & $n$ & $3 \times 2^{n-2}(n+1)$ \\
$Q(n, 1,0)$ & $Q(2,1,0)$ & $2^{n}+2^{n-2}$ & $n+2$ & $n-1$ & $5 \times 2^{n-3}(n+2)$ \\
$Q(n, 1,0)$ & $Q(3,1,0)$ & $2^{n}+2^{n-3}$ & $n+3$ & $n-1$ & $9 \times 2^{n-4}(n+3)$ \\
$Q(n, 1,0)$ & $Q(4,1,0)$ & $2^{n}+2^{n-4}$ & $n+6$ & $n-2$ & $17 \times 2^{n-5}(n+6)$ \\
$Q(n, 2,0)$ & $Q(1,2,0)$ & $2^{n}+2 \times 2^{n-1}$ & $n+2$ & $n$ & $4 \times 2^{n-2}(n+2)$ \\
$Q(n, 2,0)$ & $Q(2,2,0)$ & $2^{n}+2 \times 2^{n-2}$ & $n+3$ & $n$ & $6 \times 2^{n-3}(n+3)$ \\
$Q(n, 2,0)$ & $Q(3,2,0)$ & $2^{n}+2 \times 2^{n-3}$ & $n+5$ & $n-1$ & $10 \times 2^{n-4}(n+5)$ \\
$Q(n, 2,0)$ & $Q(4,2,0)$ & $2^{n}+2 \times 2^{n-4}$ & $n+8$ & $n-2$ & $18 \times 2^{n-5}(n+8)$ \\
$Q(n, 3,0)$ & $Q(1,3,0)$ & $2^{n}+3 \times 2^{n-1}$ & $n+3$ & $n$ & $5 \times 2^{n-2}(n+3)$ \\
$Q(n, 3,0)$ & $Q(3,3,0)$ & $2^{n}+3 \times 2^{n-3}$ & $n+7$ & $n-2$ & $11 \times 2^{n-4}(n+7)$ \\
$Q(n, 3,0)$ & $Q(4,3,0)$ & $2^{n}+3 \times 2^{n-4}$ & $n+10$ & $n-2$ & $19 \times 2^{n-5}(n+10)$ \\
\hline
\end{tabular}

\section{ONE-STEP-DEGRADABLE DESIGNS}

$Q_{n}$ can tolerate any single fault while maintaining a fault-free subgraph $Q_{n-1}$. However, two faults in antipodal positions destroy every $Q_{n-1}$ in $Q_{n}$. Figure 6 demonstrates the situation where two faulty nodes 0000 and 1111 in $Q_{4}$ destroy every $Q_{3}$. Therefore $Q_{n}$ is itself a $Q(n, 1,1)$ design. In this section, we discuss the design of $k$-FT 1-SD hypercubes by adding redundant links to the basic $Q_{n}$. Our approach is different from that in the previous section where both nodes and links are added to the underlying hypercube structures.

Let $Q_{n}^{+}$denote an $n$-dimensional hypercube with extra links. $Q_{n}^{+}$is said to be $k$-fault-tolerant g-step-degradable if $Q_{n}^{+}-F$ contains $Q_{n-g}$, where $F$ is any set of $k$ faulty nodes. A $k$-FT $g$-SD $Q_{n}^{+}$is denoted by $Q^{+}(n, k, g)$. Thus $Q^{+}(n, k, g)$ is a $Q(n, k, g)$ graph by definition. We construct $Q^{+}(n, k, 1)$ by adding a small, preferably minimum, number of extra links to $Q_{n}$. If a graph with a minimum

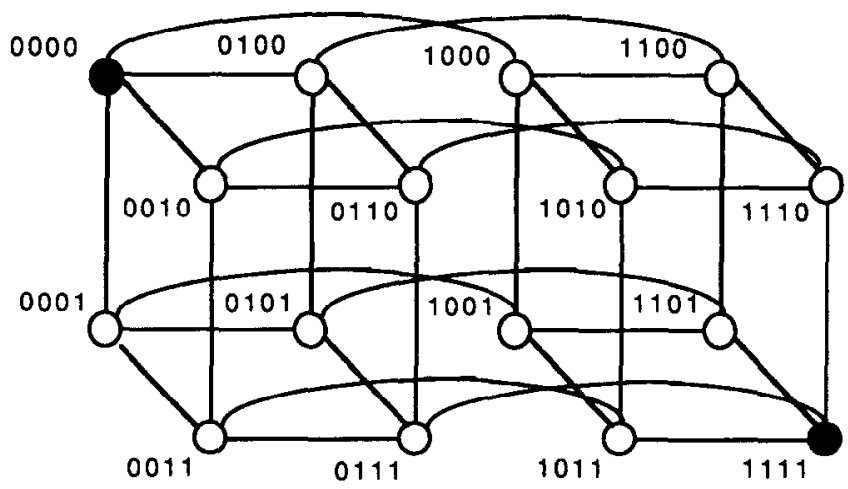

FIG. 6. Example where two node failures destroy every fault-free $Q_{3}$ in $Q_{4}$. number of links is not attainable, near-optimal solutions will be sought. Also, for practical reasons, we are interested in finding designs with low maximum node degrees.

\section{1. $Q^{+}(n, k, 1)$ Designs for $2 \leq k \leq 4$}

We start with designs with small values of $n$. The following lemma sets lower bounds for the number of links in link-optimal designs for $Q^{+}(2,2,1)$ and $Q^{+}(3,2,1)$.

Lemma 1. Link-optimal $Q^{+}(2,2,1)$ and $Q^{+}(3,2,1)$ graphs contain at least 6 and 14 links, respectively.

Proof. We prove the lemma by considering the minimum number of links that must be added to $Q_{2}$ and $Q_{3}$ to form $Q^{+}(2,2,1)$ and $Q^{+}(3,2,1)$, respectively. In each case, the number of extra links needed is greater than one. Suppose that, by way of contradiction, only one link is added, and let one of its end nodes be $x$. We can destroy every fault-free $Q_{2}$ by removing nodes $x$ and $y$, where $y$ is at distance two (three in the $Q_{3}$ case) from $x$. Hence the minimum number of extra links required is two for each of the designs. Since $Q_{2}$ and $Q_{3}$ have 4 and 12 links, respectively, the minimum numbers of links required for $Q^{+}(2,2,1)$ and $Q^{+}(3,2,1)$ are 6 and 14 , respectively.

Next, we present $Q^{+}(2,2,1)$ and $Q^{+}(3,2,1)$ with two extra links in each case. Both of these designs are linkoptimal by Lemma 1 .

THeOrem 3. The graphs $S=K_{4}$ and $W$ shown in Fig. 7 are link-optimal realizations of $Q^{+}(2,2,1)$ and $Q^{+}(3,2,1)$, respectively.

Proof. (1) $K_{4}$ clearly implements $Q^{+}(2,2,1) . K_{4}$ has two links more than $Q_{2}$; therefore, by Lemma 1 , this design is link-optimal. 


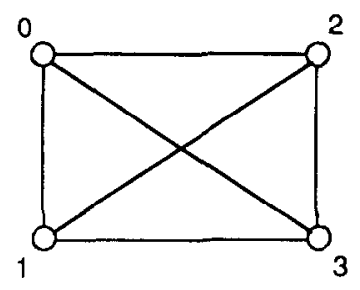

(a)

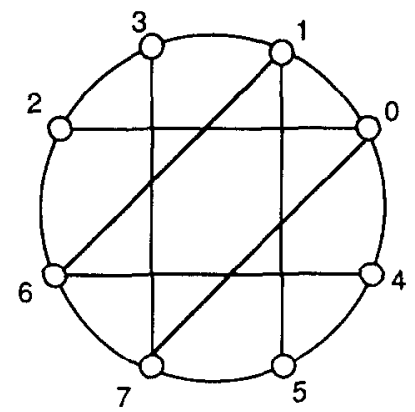

(b)
FIG. 7. (a) A link-optimal $Q^{+}(2,2,1)$ graph $S=K_{4}$. (b) A linkoptimal $Q^{+}(3,2,1)$ graph $W$.

(2) Removing from $W$ any two nodes that are distance two or less apart in the underlying $Q_{3}$ will not destroy all fault-free $Q_{2}$ 's in $W$. Hence there are four pairs of nodes, $\{0,7\},\{3,4\},\{1,6\}$, and $\{2,5\}$, whose removal may destroy each $Q_{2}$. We consider the cases for $\{0,7\}$ and $\{3,4\}$; the cases for $\{1,6\}$ and $\{2,5\}$ follow directly from symmetry. The two maximum subgraphs obtained after each node set is removed from $W$ are shown in Fig. 8; the corresponding $Q_{2}$ 's recovered are shown in heavy lines. Hence $W$ is a $Q^{+}(3,2,1)$ design. In addition, since the number of links added to the basic graph $Q_{3}$ is two, $W$ is link-optimal by Lemma 1 .

Both the $W$ and the $S$ designs have the merits of using a minimum number of links and increasing the maximum node degree by one only. $W$ is not regular, however. We next describe two regular and node-symmetric graphs that are also $Q^{+}(3,2,1)$ graphs with the same maximum node degree as $W$. These two regular graphs also reveal the fact that a degree-optimal $Q^{+}(n, k, 1)$ is not unique.

Lemma 2. Graphs $R$ and $U$ defined in Fig. 9 are degree-optimal regular realizations of $Q^{+}(3,2,1)$.

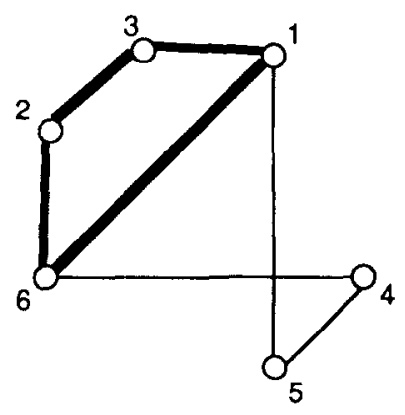

(a)

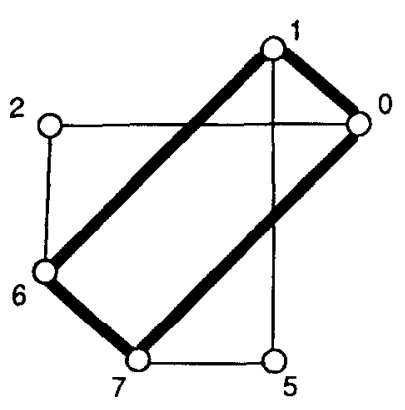

(b)
FIG. 8. The maximum subgraphs after removing node sets $\{0,7\}$ and $\{3,4\}$ from $W$. The recovered $Q_{2}$ 's are shown in heavy lines.

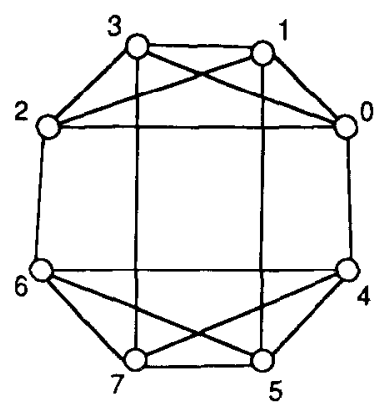

(a)

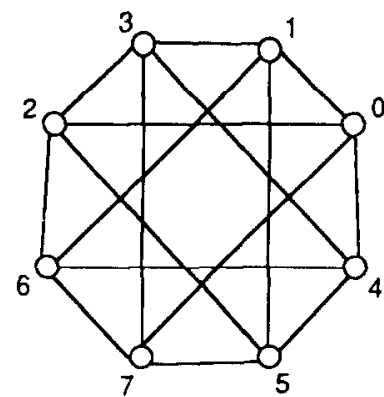

(b)
FIG. 9. Two regular degree-optimal $Q^{+}(3,2,1)$ 's (a) $R$ and (b) $U$.

Proof. $W$ (Fig. 7b) is a subgraph of $U$, hence $U$ is a $Q^{+}(3,2,1)$ graph. The proof for $R$ has two parts: First, if the two faulty nodes in $R$ are not distance three apart in the embedded $Q_{3}$, the fault-tolerance property of $Q_{3}$ guarantees the existence of a fault-free $Q_{2}$. Second, supposc that the two faulty nodes are distance three apart in the embedded $Q_{3}$. Without loss of generality, choose nodes 0 and 7 as the faulty nodes. The recovered fault-free $Q_{2}$ after nodes 0 and 7 are removed is shown in Fig. 10. The other cases follow from symmetry.

In the following, we develop the two fault-tolerant designs $B=Q^{+}(3,3,1)$ and $D=Q^{+}(3,4,1)$ shown in Fig. 11. To prove the desired fault-tolerance properties of $B$ and $D$, we need the following lemmas.

LEMMA 3. A four-node connected graph $G$ with $\delta(G) \geq 2$ is Hamiltonian.

Proof. Let the four nodes be $x_{1}, x_{2}, x_{3}$, and $x_{4}$. If $x_{1}$ connects to both $x_{2}$ and $x_{3}$, and node $x_{4}$ is connected to $x_{2}$ and $x_{3}$, then the four nodes form a cycle. Hence $G$ is Hamiltonian. Other alternatives are that $x_{4}$ is connected to $x_{2}$ and $x_{1}$, or to $x_{3}$ and $x_{1}$. Consider the case where $x_{4}$ is connected to $x_{3}$ and $x_{1}$. Then $x_{2}$ must be connected to either $x_{3}$ or $x_{4}$ since $x_{2}$ 's node degree is at least two. In either case, there is a cycle of length four in $G$; thus $G$ is Hamiltonian. The case where $x_{4}$ is connected to $x_{2}$ and $x_{1}$ follows the same reasoning.

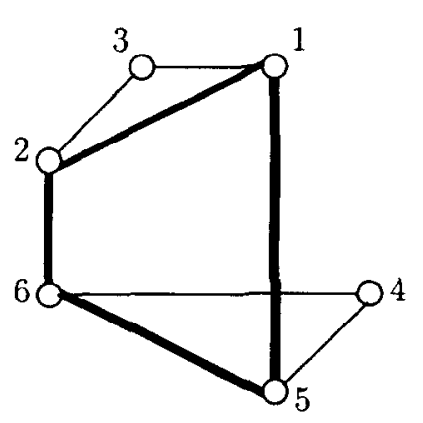

FIG. 10. The recovered $Q_{2}$ in $R$ with nodes 0 and 7 removed. 


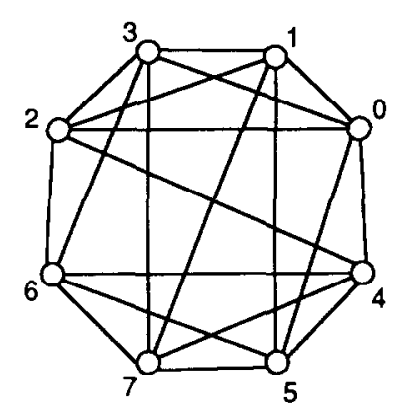

(a)

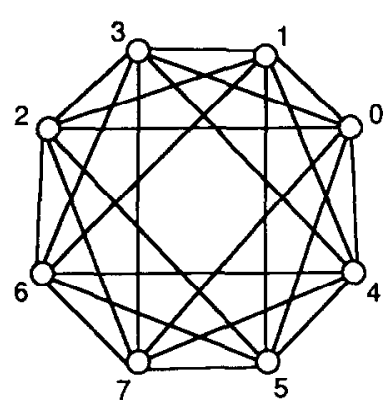

(b)
FIG. 11. (a) The $Q^{+}(3,3,1)$ graph $B$. (b) The $Q^{+}(3,4,1)$ graph $D$.

LEMMa 4. A five-node connected graph $G$ contains $Q_{2}$ if $\delta(G) \geq 2$ and at least two of its nodes have degree three or more.

Proof. Let $C_{i}$ denote a cycle with $i$ nodes. Suppose that $G$ does not contain $Q_{2}$. Then $G$ must contain either $C_{5}$ or $C_{3}$ since $\delta(G) \geq 2$. Consider the case where $G$ contains $C_{5}$. Since two nodes must have degree greater than two, at least one more link not in $C_{5}$ must exist. This links must connect two nodes of $C_{5}$ resulting in a graph containing $C_{4}=Q_{2}$, hence $G$ contains $Q_{2}$. Now consider the case where $G$ contains $C_{3}$ but not $C_{5}$. Since there are five nodes in $G$, there exist at least two $C_{3}$ 's in $G$. The intersection of two $C_{3}$ 's can include one or two nodes, depending on whether the $C_{3}$ 's share a link. When this intersection contains exactly two nodes, then the nonshared links form a $Q_{2}$. Suppose that the intersection is a single node, say $x$. The configuration is shown in Fig. 12. In this case, there is only one node of degree greater than two, hence a link must be added to the graph to meet our initial assumptions for $G$. It is obvious that adding a link between any nonadjacent nodes results in a graph containing $Q_{2}$ as a subgraph. Hence the lemma follows.

Since both $B$ and $D$ contain only eight nodes, it is easy to verify by exhaustion that there exist five (six) nodedisjoint paths between any pair of nodes in $B(D)$. By a variation of Menger's Theorem [7], $\kappa(B) \geq 5$ and $\kappa(D) \geq$

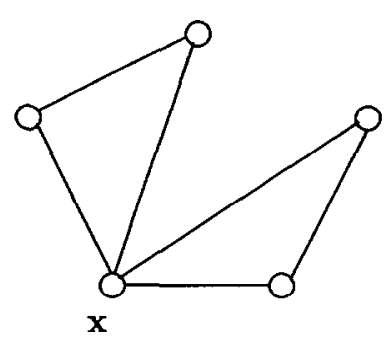

FIG. 12. Two triangles intersect at one node.
6. Therefore removing any three (four) nodes from $B(D)$ results in a connected subgraph.

Now we prove the basic fault-tolerance properties of $B$ and $D$ using Lemmas 3 and 4 .

Theorem 4. Graphs $B$ and $D$ defined in Fig. 11 realize $Q^{+}(3,3,1)$ and $Q^{+}(3,4,1)$, respectively.

\section{Proof.}

Case 1. $\delta(B)=\Delta(B)=5$. The number of links in $B$ is 20. Let $N(x)$ denote the set of neighboring nodes of $x$. We observe that for any three nodes $x, y$, and $z$ in $B, \mid N(x) \cup$ $N(y) \cup N(z) \mid \geq 7$. This implies at least two of any three nodes in $B$ are directly connected. As a result, the removal of three nodes can remove at most 14 links from $B$ and produces a graph $B^{\prime}$ which contains five nodes with no less than 6 links. Note that $\delta\left(B^{\prime}\right) \geq 2$ since $\delta(B)=5$. We now claim that at least two nodes in $B^{\prime}$ have degree three or more. Suppose that it is not the case. Then there are only three possibilities to be considered: first, the node degree of each node in $B^{\prime}$ is two; second, four nodes in $B^{\prime}$ have degree two and one node has degree three; and third, four nodes have degree two and one node has degree four. The first case is ruled out since it requires $B^{\prime}$ to have only five links. The second is also impossible since there exists a single node of odd degree. The third implies that the three removed nodes have four common neighbors in $B$. However, one can quickly verify that for any three nodes $x, y$, and $z$ in $B,|N(x) \cap N(y) \cap N(z)| \leq 3$. The third case is then impossible, hence the claim is true. By Lemma $4, B^{\prime}$ contains $Q_{2}$, and accordingly $B$ is a $Q^{+}(3,3,1)$ graph.

Case 2. $\delta(D)-\Delta(D)-6$. The removal of any four nodes from $D$ results in a four-node connected graph $D^{\prime}$ with $\delta\left(D^{\prime}\right)$ at least two. Therefore by Lemma $3, D^{\prime}$ contains $Q_{2}$.

The minimum node degree of any $Q^{+}(3,4,1)$ is six. For if the minimum node degree is less than six, we can easily remove four nodes and make the resulting four-node graph contain a node of degree less than two. In that case, it is impossible to form a $Q_{2}$. The maximum node degree of $D$ is six, hence we conclude that $D$ is degreeoptimal.

\section{2. $Q^{+}(n, k, 1)$ Designs for $5 \leq k \leq 16, n \leq 5$}

On examining the designs in the previous section, we see that a $Q^{+}(n, k+1,1)$ graph can, in general, be constructed by adding some links to a $Q^{+}(n, k, 1)$ design. For instance, the $Q^{+}(3,4,1)$ graph $D$ in Fig. $11 \mathrm{~b}$ is a supergraph of the $Q^{+}(3,3,1)$ graph $B$ in Fig. 11a, and the $Q^{+}(3,3,1)$ graph $B$ is a supergraph of the $Q^{+}(3,2,1)$ graph $R$ shown in Fig. 9. Based on this observation, we now devise a procedure to generate $Q^{+}(n, k, 1)$ for more 


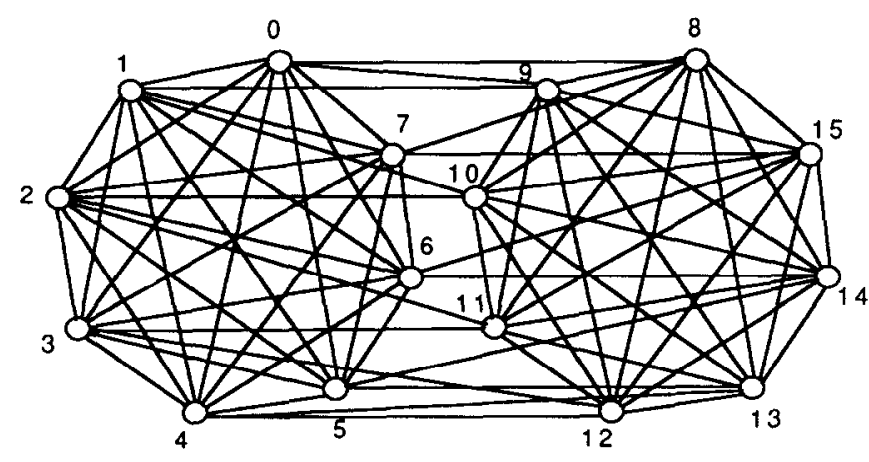

FIG. 13. A $Q^{+}(4,5,1)$ graph generated by ADD_LINKS.

general cases. The algorithm ADD_LINKS given below uses two copies of $K_{2^{n-1}}$, and adds some links between them in a systematic way. Since $K_{2^{n-1}}$ is also a $Q(n-1$, $\left.2^{n-2}, 1\right)$ graph, by connecting the corresponding nodes in the two $K_{2^{n-1}}$ 's, a $Q\left(n, 2^{n-2}, 1\right)$ is formed, which is a good starting point to construct a $Q\left(n, 2^{n-2}+l, 1\right)$, where $1 \leq l$ $\leq 2^{n-2}$.

Algorithm ADD_LINKS: $\left\{\right.$ To generate a 1-SD $Q_{n}$ graph $\left.Q\left(n, 2^{n-2}+l, 1\right)\right\}$

(1) Construct two copies of $K_{2 n-1}$, denoted $S_{1}$ and $S_{2}$. Nodes in $S_{1}$ are labeled from 0 to $2^{n-1}-1$; nodes in $S_{2}$ are labeled from $2^{n-1}$ to $2^{n}-1$.

(2) Connect each node $i$ in $S_{1}$ with all nodes $j$ in $S_{2}$, where $j \equiv i+m\left(\bmod 2^{n-1}\right)$, and $0 \leq m \leq l$.

Figure 13 demonstrates the $Q^{+}(4,5,1)$ graph generated by ADD_LINKS. Nodes $0,1,2,3,4,5,6$, and 7 form $K_{8}$, and nodes $8,9,10,11,12,13,14$, and 15 form another copy of $K_{8}$. Node 0 connects to nodes 8 and 9 , node 1 connects to nodes 9 and 10 , and so on.

The following are some properties of the interconnection between $S_{1}$ and $S_{2}$ that are useful in later proofs. Let $a$ be a node in $S_{1}$ in a given $Q(n, k, 1)$ graph, where $k=$ $2^{n-2}+l . N(a)$ is the neighboring node set of $a$ in $S_{2}$; $N N(a)$ is the fault-free neighboring node set of $a$ in $S_{2}$.

Property P1. $|N(a)|=l+1$.

Property P2. For any given i node in $S_{1}, l+i \leq$ $\left|\cup^{i} N(a)\right| \leq 2^{n-1}$.

PROPERTY P3. Suppose that there are $j$ faults in $S_{1}$ and $2^{n-2}+l-j$ faults in $S_{2}, l \leq j \leq 2^{n-2}$. For any given $i$ nonfaulty nodes in $S_{1}, 1 \leq i \leq 2^{n-2}$, we have $\left|\cup^{i} N N(a)\right|-$ $\left(i+j-2^{n-2}\right) \geq(l+i)-\left(2^{n-2}+l-j\right)-\left(i+j-2^{n-2}\right)=0$.

Properties P1 and P2 can be easily verified from the procedure itself. P3 states that for any $i$ nonfaulty nodes in $S_{1}$ that contain $j$ faults, the union of the fault-free neighboring sets of these $i$ nodes contains at least $i+j-2^{n-2}$ elements. This property can be derived from P2.
The proofs of several of our results involve some techniques from the theory of systems of distinct representatives (SDRs), which is concerned with finding a distinct representative for each set in a collection of sets. We briefly summarize here the key results we need, following the presentation of [4]. Let $B$ be an arbitrary set and let $B_{i}$ be a subset of $B$. A set $\left\{B_{1}, B_{2}, \ldots, B_{j}\right\}$ is said to have a system of distinct representatives if there exists $b_{i}, i=1$, $2, \ldots, j$, such that $b_{i}$ belongs to $B_{i}$, and $b_{i} \neq b_{j}$ if $i \neq j$.

Condition $\mathrm{C} 1$. A set $\left\{B_{1}, B_{2}, \ldots, B_{j}\right\}$ is said to satisfy Condition $\mathrm{C} 1$ if $\boldsymbol{B}_{i_{1}} \cup \boldsymbol{B}_{i_{2}} \cup \ldots \cup \boldsymbol{B}_{i_{k}}$ contains at least $k$ elements for $k=1,2, \ldots, j$, and for each choice of $i_{1}, i_{2}$, $\ldots, i_{k}$ with $1 \leq i_{1}<i_{2}<\ldots<i_{k} \leq j$.

Condition $\mathrm{C} 2$. Let $r$ be a positive integer with $r \leq j$. A set $\left\{B_{1}, B_{2}, \ldots, B_{j}\right\}$ is said to satisfy Condition $C 2$ if $B_{i_{1}} \cup$ $B_{i_{2}} \cup \ldots \cup B_{i_{k}}$ contains at least $k-(j-r)$ elements for $k=1,2, \ldots, j$, and for each choice of $i_{1}, i_{2}, \ldots, i_{k}$ with $1 \leq$ $i_{1}<i_{2}<\ldots<i_{k} \leq j$.

THEOREM 5 (Hall's Theorem). $\left\{B_{1}, B_{2}, \ldots, B_{j}\right\}$ has an $S D R$ if and only if it satisfies Condition $\mathrm{C} 1$.

Corollary 1. Let $r$ be a positive integer with $r \leq j$. Then there are $r$ sets in the family $\left\{B_{1}, B_{2}, \ldots, B_{j}\right\}$ which together have an $S D R$ if and only if $\left\{B_{1}, B_{2}, \ldots, B_{j}\right\}$ satisfies Condition $\mathrm{C} 2$.

Next we show that Algorithm ADD_LINKS can generate $Q^{+}(n, k, 1)$ correctly for $5 \leq k \leq 16$.

THEOREM 6. ADD_LINKS generates $Q^{+}(n, k, 1)$, for $5 \leq k \leq 16, n \leq 5$.

The proof of this theorem is rather lengthy and can be found in [9]. We sketch the proof for a representative case $(k=10)$ here to illustrate how ADD_LINKS is able to generate $Q^{+}(5,10,1)$. In this case, the generated $Q(5,10,1)$ contains two copies $S_{1}$ and $S_{2}$ of $K_{16}$. Each node $x$ in $S_{1}$ connects to three nodes in $S_{2}$ and vice versa. Since a $Q(5,10,1)$ graph constructed this way always contains $Q(5,9,1)$ as subgraph, we will focus on the cases where there are 10 faults in the system.

Suppose that all 10 faults lie either in $S_{1}$ or else in $S_{2}$. Since $S_{1}$ and $S_{2}$ are copies of $K_{16}$, it is clear that either $S_{2}$ or $S_{1}$ should contain a fault-free $Q_{4}$ in this case. Now suppose nine faults are in $S_{1}$ and one in $S_{2}$. We can find one nonfaulty node $a$ in $S_{1}$ which connects to three nonfaulty nodes in $S_{2}$. The subgraph induced by node $a$ and the nonfaulty nodes in $S_{2}$ contains a fault-free $Q_{4}$.

Next, suppose there are $j$ faults in $S_{1}$ and $10-j$ faults in $S_{2}, j \geq 2$. Let $a$ denote a fault-free node in $S_{1}$. By Property P3 above, $\left|\bigcup^{i} N N(a)\right|-(i+10-8)=$ $\left|\cup^{i} N N(a)\right|-i+2 \geq 0$, for any given $i$ nonfaulty nodes in $S_{1}, 1 \leq i \leq 16-j$. Therefore by Corollary 1 , we can find eight nonfaulty nodes in $S_{1}$ each of which has a distinct nonfaulty neighbor in $S_{2}$. The graph induced by these 
TABLE II

The Increase in the Node Degree in the $Q(n, k, 1)$ Graphs Generated by ADD_LINKS, $2 \leq k \leq 16$

$\begin{array}{llllllllllllllll}\text { Fault tolerance } k: & 2 & 3 & 4 & 5 & 6 & 7 & 8 & 9 & 10 & 11 & 12 & 13 & 14 & 15 & 16\end{array}$

$\begin{array}{llllllllllllllll}\text { Increased degree: } & 1 & 2 & 3 & 5 & 6 & 7 & 8 & 12 & 13 & 14 & 15 & 16 & 17 & 18 & 19\end{array}$

nodes and their distinct nonfaulty neighbors contains a fault-free $Q_{4}$. The cases for other fault patterns follow by symmetry.

ADD_LINKS is not limited to generating the design specified in Theorem 6 ; it can also generate $Q^{+}(n, k, 1)$ for some larger $k$ 's, as stated in the next theorem. This leads us to the conjecture that an ADD_LINKS-like algorithm can generate $Q^{+}(n, k, 1)$ for arbitrary $k$.

THEOREM 7. ADD_LINKS generates $Q^{+}\left(n, 2^{n-2}+1\right.$, 1), $n \geq 2$.

\section{Proof.}

Case 1. There are no more than $2^{n-2}$ faults. The graph created by ADD_LINKS contains $K_{2} \times K_{2}^{n}$, which is a realization of $Q^{+}\left(n, 2^{n-2}, 1\right)$. Therefore if the number of faults does not exceed $2^{n-2}$, a fault-free $Q_{n-1}$ is present.

Case 2. There are $2^{n-2}+1$ faults, all of which lie in either $S_{1}$ or $S_{2}$. In this case, $S_{2}$ or $S_{1}$ contains a fault-free $Q_{4}$.

Case 3. There are jaults in $S_{1}$ and $2^{n-2}+l-j$ faults in $S_{2}$. Given $i$ nonfaulty nodes in $S_{1}$, by Property P3, $\left|\cup^{i} N N(a)\right|-\left(i+k-2^{n-2}\right) \leq 0$. By Corollary 1 , we can find $2^{n-2}$ nonfaulty nodes in $S_{1}$, each of which has a nonfaulty distinct neighboring node in $S_{2}$. The $2^{n-2}$ nodes in $S_{1}$ form a $Q_{n-2}$ and so do their corresponding nonfaulty neighbors in $S_{2}$. Together, these two $Q_{n-2}$ 's form a faultfree $Q_{n-1}$. All other cases follow by symmetry.

The node degree of the $Q(n, k, 1)$ graphs generated by this procedure is $n+\left(2^{d}+k-d-2\right)$, where $2^{d}<k \leq$ $2^{d+1}$. This node degree increases by $2^{d}+k-d-2$ compared to $Q_{n}$. Table II lists the increase in the node degree of the $Q(n, k, 1)$ graphs, where $2 \leq k \leq 16$. It shows that the node degree increases at a moderate rate with the fault-tolerance parameter $k$.

\section{3. $Q^{+}(n, k, 1)$ Designs for Large $n$}

The discussion so far has focused on $Q^{+}(n, k, 1)$ for small $n$, e.g., $n \leq 5$. As in the $Q(n, k, 0)$ case, these smaller designs can be used to construct larger ones by the product method, as characterized in the ncxt corollary. The proof of this result is similar to that of Theorem 2 and thus omitted.

CoRollary 2. $G^{\prime}=K_{2} \times G$ is a $Q^{+}(n, k, 1)$ graph if and only if $G$ is $a Q^{+}(n-1, k, 1)$ graph, for $n, k \geq 2$.
The graph-product construction preserves the faulttolerance property of the seed graph. Suppose that $G \in$ $\{R, U, B, D\}$; we define $G_{n}$ recursively as follows: $G_{3}=$ $G$, and $G_{n+1}=K_{2} \times G_{n}$. By Corollary 2, Lemma 2, and Theorem $4, R_{n}, U_{n}, B_{n}$, and $D_{n}$ are $Q^{+}(n, 2,1)$, $Q^{+}(n, 2,1), Q^{+}(n, 3,1)$, and $Q^{+}(n, 4,1)$ graphs, respectively. They are illustrated for $n=4$ in Fig. 14. A great advantage of this construction method is that the increase in the node degree of a design compared to $Q_{n}$ is the same
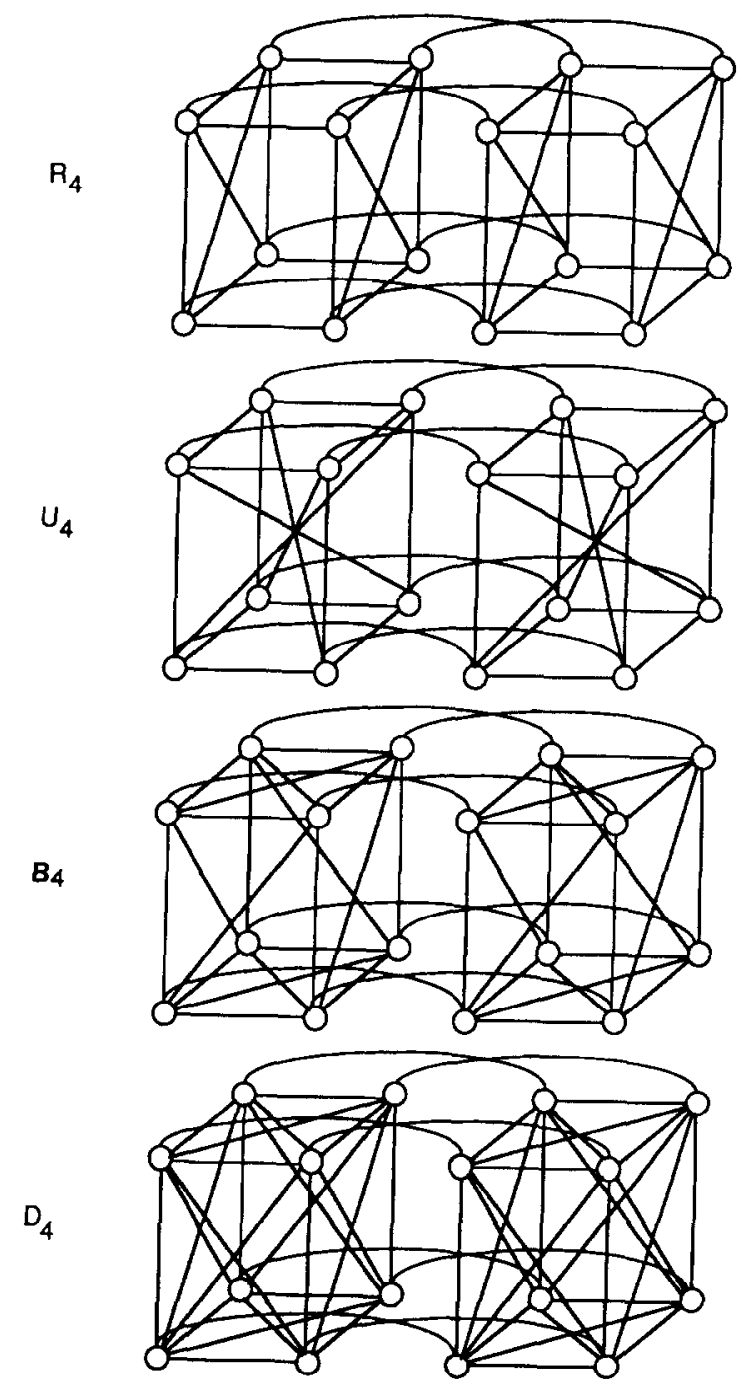

FIG. 14. Examples of $Q^{+}(4, k, 1)$ graphs: $R_{4}$ and $U_{4}$ are $Q^{+}(4,2,1)$ designs; $B_{4}$ and $D_{4}$ are $Q^{+}(4,3,1)$ and $Q^{+}(4,4,1)$ designs, respectively. 


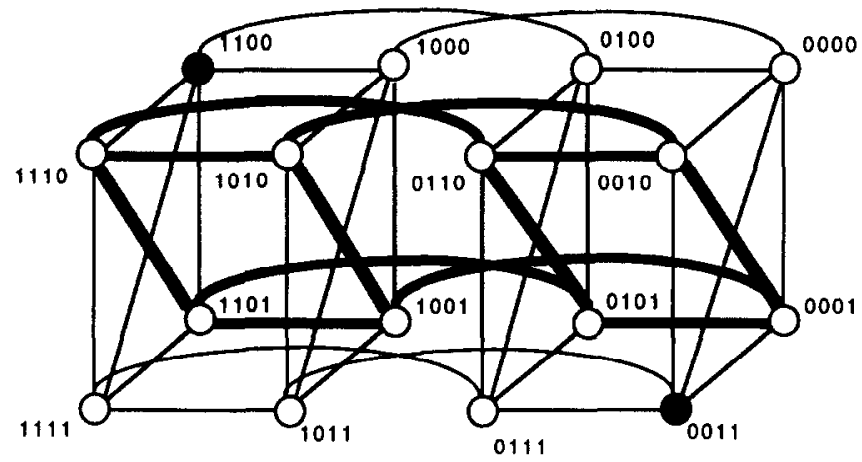

FIG. 15. The recovered $Q_{3}$ (heavy lines) in a faulty $R_{4}$.

as that of its seed. For instance, the node degree of $B_{4}$ is six. The increase in the node degree is two compared to $Q_{4}$, and is the same as the node degree increase of $B_{3}$, its seed, compared to $Q_{3}$.

Designs of this form can be easily reconfigured when faults occur. Consider the case of the $Q^{+}(4,2,1)$ graph $R_{4}$ which appears in Fig. 15. Suppose that two nodes 0011 and 1100 are faulty. The subcube $0^{* * *}$ is a $Q^{+}(3,2,1)$ seed. We can project the faults into the sced to obtain a pseudo-fault set $F^{\prime}=\{0011,0100\}$. Since the seed is relatively small, it is simple to recover a fault-free $Q_{2}$. In this specific example, it is easy to find the four pseudo-faultfree nodes $0110,0101,0001$, and 0010 which form a pseudo-fault-free $Q_{2}$. The neighbors of these four nodes in the other seed $1^{* * *}$ are also pseudo-fault-free. Together they form a fault-free $Q_{3}$, which is shown in Fig. 15 by heavy lines.

\subsection{Topological Properties}

The spare links added to $Q_{n}$ to construct $Q^{+}(n, k, 1)$ not only increase fault tolerance with respect to $Q_{n-1}$, but also improve some useful topological properties of $Q_{n}$. These links reduce the diameter and shorten the average internode distance of the graph. They also allow some useful graphs to be embedded compactly in the proposed structures.

The topological characteristics of the proposed designs are summarized and compared with those of $Q_{n}$ in Table
III. It is easily seen that if the diameter of $G$ is $d$, then the diameter of $G^{\prime}=K_{2} \times G$ is $d+1$. Using this property together with the observation that the diameter of the graphs $R, U, B$, and $D$ is two, the diameters of $R_{n}, U_{n}, B_{n}$, and $D_{n}$ can be shown to be $n-1$. The number of links in each graph can be determined from the following result [7]. If $G_{1}=\left(V_{1}, E_{1}\right)$ and $G_{2}=\left(V_{2}, E_{2}\right)$, then the number of links in graph $G_{1} \times G_{2}$ is $\left|V_{1}\right|\left|E_{2}\right|+\left|V_{2}\right|\left|E_{1}\right|$.

The link overhead of a fault-tolerant design $Q_{n}^{+}$is defined to be the ratio of the number of redundant links in $Q_{n}^{+}$to the number of links in the underlying $Q_{n}$. Table III shows that the link overhead in each of our designs is small. For instance, the link overhead is $1 / n$ in $R_{n}$ and $U_{n}$; it is $5 / 2 n$ in $B_{n}$ and $3 / n$ in $D_{n}$.

Let $d\left(v_{1}, v_{2}\right)$ be the distance between nodes $v_{1}$ and $v_{2}$. The average distance of $G=(V, E)$ is defined as

$$
\frac{\sum_{1 \leq i \leq j \leq V \mid} d\left(v_{i}, v_{j}\right)}{\left(\begin{array}{c}
V \mid \\
2
\end{array}\right)} .
$$

The distance parameters of the proposed $Q^{+}(n, k, 1)$ designs are also listed in Table III for comparison with $Q_{n}$. Note that with a small increase in the number of links and the node degrees, the fault-tolerance capabilities of the proposed structures are increased, while the average internode distance is decreased.

The proposed structures embed more graphs than $Q_{n}$. Any cubical graph can also be embedded in the four $Q^{+}(n, k, 1)$ graphs, since $Q_{n}$ is a subgraph of $R_{n}, U_{n}, B_{n}$, and $D_{n}$. Furthermore, $R_{n}$ can embed a cycle $C_{i}$ for any $i$, while $Q_{n}$ can only embed $C_{i}$ for even $i$ 's. $B_{n}$ and $D_{n}$ have this property as well, since each contains $R_{n}$ as a subgraph. In addition, $T_{n}$, the full binary tree of height $n$, can be embedded in $R_{n}, B_{n}$, and $D_{n}$ more compactly than in hypercubes. It is known that $T_{n}$ can be embedded in $Q_{n+2}$ but not in $Q_{n+1}$. Nebesky [10] defines $Q_{n}^{\nabla}$ to be the graph $Q_{n}+r t-s$, where $r, s$, and $t$ are nodes of $Q_{n}$ such that $r s$ and $s t$ are distinct edges of $Q_{n}$. He then shows that $T_{n}$ is a spanning subgraph of $Q_{n+1}^{\nabla}$, for $n \geq 1$. Since $Q_{n+1}^{\nabla}$ is a subgraph of $R_{n+1}, T_{n}$ can be embedded in $R_{n+1}$, and therefore also in $B_{n+1}$ and $D_{n+1}$.

TABLE III

Topological Properties of $Q_{n}, R_{n}, B_{n}$, and $D_{n}$

\begin{tabular}{ccccccc}
\hline Graphs & $\begin{array}{c}\text { Fault } \\
\text { tolerance }\end{array}$ & $\begin{array}{c}\text { Number } \\
\text { of nodes }\end{array}$ & $\begin{array}{c}\text { Number } \\
\text { of links }\end{array}$ & $\begin{array}{c}\text { Maximum } \\
\text { node degree }\end{array}$ & Diameter & $\begin{array}{c}\text { Average } \\
\text { distance }\end{array}$ \\
\hline$Q_{n}$ & $Q^{+}(n, 1,1)$ & $2^{n}$ & $n 2^{n-1}$ & $n$ & $n$ & $\approx n / 2$ \\
$R_{n}$ & $Q^{+}(n, 2,1)$ & $2^{n}$ & $(n+1) 2^{n-1}$ & $n+1$ & $n-1$ & $\approx n / 2-1 / 4$ \\
$B_{n}$ & $Q^{+}(n, 3,1)$ & $2^{n}$ & $(n+2) 2^{n-1}$ & $n+2$ & $n-1$ & $\approx n / 2-1 / 2$ \\
$D_{n}$ & $Q^{+}(n, 4,1)$ & $2^{n}$ & $(n+3) 2^{n-1}$ & $n+3$ & $n-1$ & $\approx n / 2-5 / 8$ \\
\hline
\end{tabular}




\section{TWO-STEP-DEGRADABLE DESIGNS}

We show in this section that the proposed $Q^{+}(n, k, 1)$ 's can serve as 2-SD hypercubes $Q^{+}(n, k, 2)$ with high levels of fault tolerance. To facilitate the discussion, we use $R_{3}, W_{3}$, and $D_{3}$ to denote the $Q(3,2,1), Q(3,3,1)$, and $Q(3,4,1)$ graphs presented previously; see Figs. 9 and 11 . The $Q(n, k, 1)$ graphs derived from these graphs using the product method are termed $R_{n}, W_{n}$, and $D_{n}$.

To begin, we demonstrate the 2-SD fault-tolerance property of $R_{3}, B_{3}$, and $D_{3}$. It is not hard to see that $R_{3}$, $B_{3}$, and $D_{3}$ can tolerate up to five faults, while a fault-free subgraph $Q_{2}$ exists. The reason is that each of them contains two copies of $K_{4}$. It takes three faults to destroy a fault-free $Q_{1}$ in $K_{4}$. Therefore it takes at least six faults to destroy every fault-free $Q_{1}$. Fault sets that destroy every fault-free $Q_{1}$ in $R_{3}, B_{3}$, and $D_{3}$ are shown in Fig. 16. Therefore $R_{3}, B_{3}$, and $D_{3}$ are $Q(3,5,2)$ graphs.

In general, it is not easy to determine the number of faults that a $Q(n, k, 1)$ graph can tolerate while allowing a $Q_{n-2}$ to be recovered. However, if $Q(n, k, 1)$ is constructed by the graph-product method, a lower bound on this number can be obtained, which is characterized in the next theorem.

THEOREM 8. If $G_{n}$ is a $Q(n, k, 1)$ graph, then $K_{2} \times G_{n}$ is a $Q(n+1,2 k+1,2)$ graph $G_{n+1}$.

The theorem can be explained as follows. $G_{n+1}$ consists of two disjoint copies of $G_{n}$. Since $G_{n}$ is a $Q(n, k, 1)$

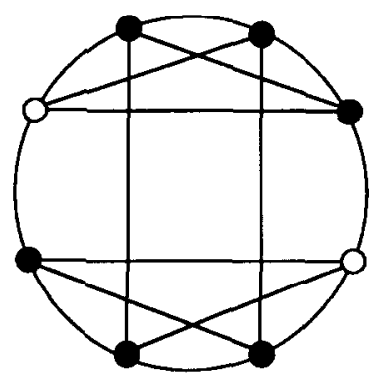

(a)

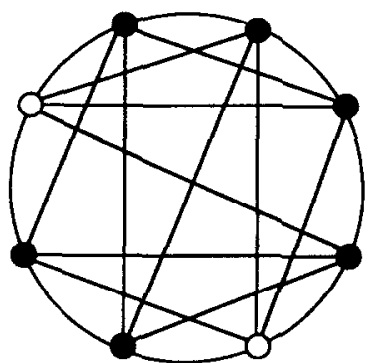

(b)

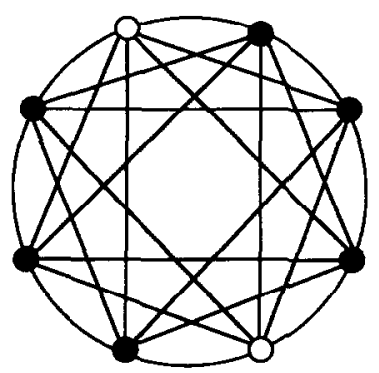

(c)
FIG. 16. Faults sets that destroy every fault-free $Q_{1}$ in (a) $R_{3}$, (b) $B_{3}$, and (c) $D_{3}$.

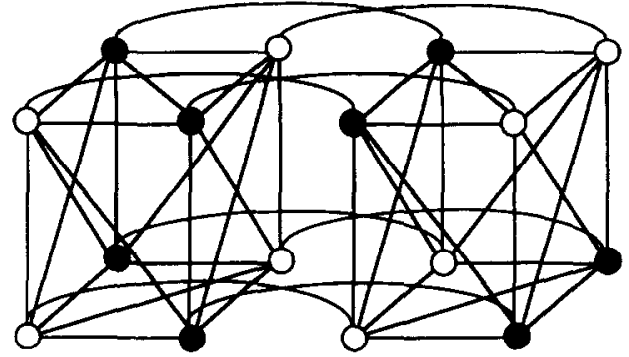

(a)

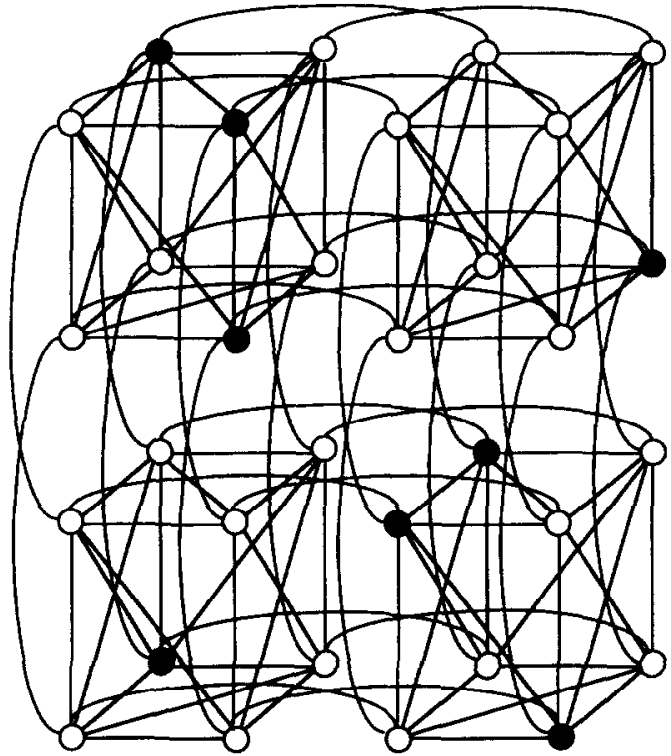

(b)

FIG. 17. Fault sets that destroy every fault-free $Q_{2}$ and $Q_{3}$ in (a) $W_{4}$ and (b) $W_{5}$, respectively.

graph, it takes at least $k+1$ faults to destroy every faultfree $Q_{n-1}$ in each copy of $G_{n}$. It takes at least $2(k+1)$ faults to destroy every fault-free $Q_{n-1}$ in $G_{n+1}$. Thus $G_{n+1}$ can tolerate at least $2 k+1$ faults while a fault-free subgraph $G_{n-1}$ exists. Therefore $G_{n+1}$ is a $Q(n+1,2 k+1,2)$ graph.

It is not clear, however, whether $2 k+1$ faults is the maximum number that $G_{n+1}$ can tolerate while a fault-free $Q_{n-1}$ is present. Nevertheless, in some special cases, $2 k+1$ can be proved to be the maximum by explicitly constructing the fault set. For instance, Fig. 17 demonstrates how eight faults can destroy every fault-free $Q_{2}$ and $Q_{3}$ in $W_{4}$ and $W_{5}$, respectively.

Theorem 8 implies that the $Q^{+}(n, k, 1)$ designs can be taken as two-step-degradable structures with a high level of fault tolerance. For example, it takes at least 8, 9, and 10 faults to destroy every fault-free $Q_{n-2}$ in $R_{n}, W_{n}$, and $D_{n}$. Many of the advantages of our previous designs, such as low increase in node degree and ease of reconfiguration, also apply to such designs. We list in Table IV the 
TABLE IV

The Fault Tolerance of the $Q(n, k, 1)$ Designs with Respect to $Q_{n-2}$

\begin{tabular}{cc}
\hline & $\begin{array}{c}\text { Fault tolerance } \\
\text { with respect to } \\
\text { Graph }\end{array}$ \\
\hline $\begin{array}{c}Q_{n-2} \\
Q(n, 2,1)\end{array}$ \\
$Q(n, 3,1)$ & $\geq 5$ \\
$Q(n, 4,1)$ & $\geq 7$ \\
$Q(n, 5,1)$ & $\geq 9$ \\
$Q(n, 6,1)$ & $\geq 13$ \\
$Q(n, 7,1)$ & $\geq 15$ \\
$Q(n, 8,1)$ & $\geq 17$ \\
$Q(n, 9,1)$ & $\geq 19$ \\
$Q(n, 10,1)$ & $\geq 21$ \\
\hline
\end{tabular}

corresponding fault tolerance of the $Q(n, k, 1)$ designs used as two-step-degradable structures.

\section{CONCLUSIONS}

We have presented a novel characterization of faulttolerant hypercube structures that allows the performance degradation due to faults to be quantified. We developed specific fault-tolerant designs for zero-, one-, and two-step degradation. We showed that by using a graph-product construction, a large fault-tolerant design can be developed using a small design as a seed. Most of our designs are regular and node-symmetric, and are quite efficient in terms of the overhead associated with the spare links and the maximum node degree.

The graph-product construction technique can be extended to other systems defined by product graphs. Suppose that $G=G_{1} \times G_{2}$, and $G_{1}^{\prime}$ is a $k$-FT realization of $G_{1}$. Then $G^{\prime}=G_{1}^{\prime} \times G_{2}$ is a $k$-FT realization of $G$. We can illustrate this for an $m \times n$ mesh $M=P_{m} \times P_{n}$. $C_{m+1}$, a loop with $m+1$ nodes, is a 1-FT realization of $P_{m}$. $M^{\prime}=$ $C_{m+1} \times P_{n}$ is then a $1-F T$ realization of $M$. This method provides a way to construct general fault-tolerant designs efficiently and systematically.

\section{REFERENCES}

1. Banerjee, P., Rahmeh, J., Stunkel, C., Nair, S., Roy, K., and Abraham, J. An evaluation of system-level fault tolerance on the
Intel hypercube multiprocessor. Proc. 18th Symposium on FaultTolerant Computing, 1988, pp. 362-367.

2. Becker, B., and Simon, H. U. How robust is the $n$-cube? Proc. $27 t h$ Annual Symposium on Foundations of Computer Science, 1986, pp. 283-291.

3. Bhuyan, L. N., and Agrawal, D. P. Generalized hypercube and hypercube structures for a computer network. IEEE Trans. Comput. 33 (Apr. 1984), 323-333.

4. Brualdi, R. A. Introductory Combinatorics. North-Holland, New York, 1983.

5. Dutt, S., and Hayes, J. P. An automorphic approach to the design of fault-tolerant multiprocessors. Proc. 19th Symposium on FaultTolerant Computing, 1989, pp. 496-503.

6. Graham, N., Harary, F., Livingston, M., and Stout, Q. F. Subcube fault-tolerance in hypercubes. Inform. and Comput., to appear.

7. Harary, F. Graph Theory. Addison-Wesley, Reading, MA, 1969.

8. Krishnamoorthy, M. S., and Krishnamurthy, B. Fault diameter of interconnection network, Comput. Math. Appl. 13, 5/6 (1987), 577582.

9. Lee, T. C. Design of fault-tolerant hypercube computers. Ph.D. thesis, University of Michigan, Ann Arbor, MI, 1990.

10. Nebesky, L. On cubes and dichotomic trees. Časopis Pèst. Mat. 99 (1974), 164-167.

11. Rennels, D. A. On implementing fault-tolerance in binary hypercubes. Proc. 16th Symposium on Fault-Tolerant Computing, 1986, pp. 344-349.

12. Yanney, R. M. Fault recovery in multiprocessor networks. Ph.D. thesis, University of Southern California, Los Angeles, CA, 1982.

TZE CHIANG LEE received the B.S. degree in electrical engineering from the National Taiwan University in 1982. He received the M.S. degree in computer information and control engineering and the Ph.D. degree in computer science and engineering from the University of Michigan in 1988 and 1990, respectively. He is currently a research staff member at IBM Thomas J. Watson Research Center, Yorktown Heights, New York. His research interests include performance evaluation, computer architecture, parallel and distributed systems, and faulttolerant computing. Dr. Lee is a member of the IEEE Computer Society and the ACM.

JOHN P. HAYES received the B.E. degree from the National University of Ireland in 1965, and the M.S. and Ph.D. degrees from the University of Illinois in 1967 and 1970, respectively, all in electrical engineering. He has been a professor in the EFCS Department at the University of Michigan since 1982. He teaches and conducts research in the areas of computer-aided design and testing, computer architecture, and fault-tolerant computing. Hayes has written several books including Computer Architecture and Organization (2nd ed., McGraw-Hill, 1988) and Hierarchical Modeling for VLSI Circuit Testing (Kluwer, 1990 , coauthored with D. Bhattacharya), as well as over a hundred technical papers. He is a fellow of IEEE and a member of ACM and Sigma Xi. 